\title{
La enseñanza de la autorregulación en aulas de educación primaria
}

Teaching self-regulation in classrooms of elementary school

Ensinar autorregulação nas aulas de ensino fundamental

\section{DOI: https://doi.org/10.18861/cied.2021.12.2.3055}

\section{Valentina Ronqui}

Universidad Católica del Uruguay

Uruguay

valeronqui@gmail.com

Maria Fernanda Sánchez

Universidad Católica del Uruguay

Uruguay

fernandasanch@gmail.com

ORCID: 0000-0002-4237-0769

\section{Daniel Trías}

Universidad Católica del Uruguay

Uruguay

ORCID: 0000-0003-1274-9134

\section{Recibido: $14 / 12 / 20$}

Aprobado: 15/03/2

Cómo citar: Ronqui, V., Sánchez, M. F.. \& Trías, D. (2021). La enseñanza de la autorregulación en aulas de educación primaria. Cuadernos de Investigación Educativa, 12(2). https://doi.org/10.18861/cied.2021.12.2.3055

\section{Resumen}

La ensenanza explicita de la autorregulacion puede contribuir al mejoramiento de la comprensión de textos, aunque no parece tan extendida su implementación efectiva en las aulas de educación primaria. El objetivo es evaluar la incidencia de la enseñanza de sestrateglas de autorregulacion del aprendizaje en la comprensión de textos escritos, mediante una intervención educativa implementada en el aula, para apoyar el aprendizaje académico que realizan escolares de quinto año a partir de textos en el area de cienclas sociales. Se espera determinar los efectos de la intervencion tambien en los estudiantes con diagnóstico de dislexia. Participaron 69 escolares de 5. año de un colegio de nivel socioeconomico medio-alto de Montevideo, y 20 de ellos presentaban diagnostico de dislexia. Se realizo un estudio cuasi experimenta de una maestra enfocada en la enseñanza de estrategias de aprendizaje autorregulado en la comprensión lectora de textos informativos, y distribuida en doce sesiones de una hora cada una Se administraron pruebas de comprensión de textos, autorregulación del aprendizaje y eficacia lectora En las distintas comparaciones se utilizó la prueba ANCOVA así como estadísticos no paramétricos Los participantes en la condición experimental aumentaron significativamente su puntuación en comprensión de textos y en autorregulación. En los niños con diagnóstico de dislexia del grupo experimental se observó un aumento significativo con respecto a su puntuación en autorregulación. Si bien la intervención en el aula beneficia al conjunto de los estudiantes, se discute sobre los efectos diferenciales de la enseñanza de la autorregulación. Las caracteristicas de la intervención vuelven factible su implementación en el aula de educación primaria a la hora de abordar la comprension y el aprendizaje a partir de textos.

Palabras clave: comprensión lectora, aprendizaje autorregulado, metacognición, estrategias, enseñanza.

\section{Abstract}

The explicit teaching of self-regulation can contribute to improve text comprehension, although th effective implementation in classrooms of elementary schools seems to be scarcely extended n learning regarding the comprehension of written texts through educational intervention to be mplemented in classrooms, aimed at supporting the academic learning by elementary students effects also for students diagnosed with dyslexia. Sixty nine fifth-grade students from an elementary of which 20 had been diagnosed with dyslexia A quasi-experimental study was carried out with prepost measures and a quasi-control group. The experimental group participated in an intervention in a classroom carried out by a teacher who focused on teaching strategies of self-regulated learning for reading comprehension of information texts. Said intervention was distributed in twelve sessions were applied The ANCOVA test and non-parametric statistics were used for different comparisons Participants in this experimentatcondtion increased significantly their scores fortext comprehension and self-regulation. As for children diagnosed with dyslexia in the experimental group a significant avorable for all the group of students, the differential effects of self-regulation teaching are under discussion. The characte

Keywords: reading comprehension, self-regulated learning metacognition, strategies, teaching

\section{Resumo}

D ensino explicito da autorregulação pode contribuir no melhoramento da compreensão textual. embora não pareça extensiva sua efetiva implementaçao nas aulas de ensino fundamental. O objetivo e avaliar a incidencia do ensino de estratéglas de autorregulaçáa da aprenaizagemn na compreensáo de sociais. Confia-se também determinar os efeitos da intervencão em alunos com diagnóstico de dislexia Participaram alto de Montevidéu, dos quais 20 tinham diagnóstico de dislexia. Uma pesquisa quase experimental oi realizada com medidas pre-pos e um grupo quase-controle. O grupo experimental participou de uma intervençáo realizada por uma professora na sala de aula, voltada para o ensino de estrateglas de aprendizagem autorregulado na compreensao leitora de textos informativos, e distribuida en da aprendizagem e eficiencia na leitura Foram usados o teste ANCOVA a a estatistica não paramétrica nas diferentes comparacões Os participantes na condicão experimental aumentaram significativamente suas pontuacões em compreensão de texto e autorregulação. Nas crianças com diagnóstico de dislexia do grupo experimental, fol observado um aumento significativo no escore de autorregulaçăo. Embora a intervençăo em sala de aula beneficie todos os alunos, discute-se sobre os efeitos diferencialis do ensino da autorregulaçaó. As caracteristicas da intervençăo permitem endizagem de textos 


\section{Introducción}

La enseñanza de la autorregulación del aprendizaje constituye un desafio en distintos niveles del sistema educativo, asi como para quienes investigan en la temática. Los distintos sistemas educativos del mundo buscan promover aprendizajes flexibles que permitan seguir aprendiendo a lo largo de la vida (UNESCO, 2016). En Uruguay también ha ido creciendo el interés por el desarrollo de las habilidades socioemocionales y, particularmente, la autorregulación en el aprendizaje (INEEd, 2018). La enseñanza de la autorregulación cuestiona, con vigencia, sobre las experiencias y las ayudas instruccionales que contribuyen a su desarrollo (Alexander, 2018; Zimmerman, 2013).

Desde la investigación en psicología educativa se concibe a la autorregulación del aprendizaje como un conjunto de procesos psicológicos por los cuales las personas gobiernan sistémicamente sus propios pensamientos, sentimientos y acciones para alcanzar sus objetivos en situaciones académicas (Zeidner y Stoeger, 2019). La autorregulación del aprendizaje es considerada una variable moduladora que tiene incidencia sobre el desempeño de los estudiantes en distintos niveles del sistema educativo (Dent y Koenka, 2016).

Existen distintos modelos teóricos que dan cuenta de los procesos implicados en la autorregulación y de sus relaciones entre sí, entre los cuales el modelo cíclico de Zimmerman es el de uso más extendido en la investigación educativa (Greene, 2018). Este modelo se enfoca particularmente en aspectos cognitivos y metacognitivos, y concibe a la autorregulación como un ciclo recurrente de las fases de planificación, ejecución y autorreflexión. En cada fase se despliega una serie de subprocesos tales como definición de metas, análisis de tareas, autoeficacia, monitoreo, reflexión sobre el proceso, autorreacciones (Zimmerman, 2013). Sobre esta base teórica se han explorado las posibilidades de enseñanza de la autorregulación en distintos dominios tales como la comprensión de textos, las matemáticas o el aprendizaje de las ciencias (Panadero, 2017).

\section{Autorregulación del aprendizaje y comprensión de textos}

El interés por las estrategias de autorregulación y su enseñanza se vincula a uno de los aprendizajes que ocupa a los sistemas educativos del mundo como es el de comprensión de textos. Aprender a comprender textos es crucial para seguir aprendiendo y es una de las formas privilegiadas por el sistema educativo para acceder al conocimiento (Kim, 2015). Comprender y aprender a partir de los textos implica, entre otras cosas, el desarrollo de estrategias de autorregulación de aspectos cognitivos, metacognitivos y afectivo-motivacionales. En Uruguay, el informe Aristas 2017 (INEEd, 2018) concluye que, en lo que respecta a comprensión de textos, casi la mitad de los estudiantes de sexto año de educación primaria no logra generalizar informaciones implicitas complejas ni extraer conclusiones a partir de argumentos que involucran una lectura global. 
Con el foco en las estrategias de autorregulación se asume que el monitoreo y la regulación del proceso lector tienen efectos significativos en la comprensión de textos (Steiner, van Loon, Bayard y Roebers, 2020). De hecho, el nivel de dominio de estrategias vinculadas a la planificación, ejecución y evaluación de la actividad lectora podrían explicar en gran medida las diferencias entre los peores y mejores lectores (Fonseca et al., 2014). Por tanto, el desarrollo de estrategias de autorregulación contribuye a niveles más profundos de comprensión y puede ser aún más necesario en contextos virtuales (Burin, González, Barreyro e InjoqueRicle, 2020).

Para lograr un manejo significativo del contenido de un texto deben activarse diversos procesos que requieren enseñanza (Gaeta, 2015). La investigación acumulada muestra que la enseñanza explícita de estrategias de autorregulación puede contribuir positivamente al aprendizaje en distintos dominios y niveles educativos (de Boer, Donker, Kostons y van der Werf, 2018; Dignath y Büttner, 2008; Perry, Lundie y Golder, 2018). Para ello la enseñanza de estrategias debería ser explícita: combinar estrategias cognitivas, metacognitivas y volitivas, y ajustarse a las necesidades de la tarea (Dignath y Büttner, 2008). En particular, el efecto positivo de la enseñanza de estrategias se observa en el aprendizaje de la lectura y la escritura en educación primaria (Dignath, Buettner y Langfeldt, 2008; Okkinga et al., 2018). Por tanto, la enseñanza explicita de estrategias de aprendizaje autorregulado puede ser una vía para mejorar la habilidad para comprender textos y aprender a partir de los mismos, que tanto se valora.

En un metaanálisis que reúne 52 estudios en contexto de aula, Okkinga et al. (2018) recogen los efectos positivos de la enseñanza de estrategias. Gracias a las intervenciones, entre sus conclusiones destacan que los alumnos de entre 8 y 14 años de edad ven incrementado su desempeño en cuanto a la comprensión de textos, lo que sugiere la importancia de la enseñanza explícita de estrategias en esa etapa. Beneficios similares se observan en estudiantes con dificultades de aprendizaje (Sanders et al., 2019; Ritchey et al., 2017). En su estudio de metaanálisis Sanders et al. (2019) encuentran que la mayor parte de los estudios con niños con dificultades de aprendizaje se realizan en contextos más controlados y abogan por más estudios que consideren la enseñanza impartida por docentes en el contexto del aula.

\section{Las estrategias de autorregulación en el aula}

Por una parte, si bien se constatan efectos positivos de la enseñanza explicita de la autorregulación en comprensión de textos, no parece tan extendida su implementación efectiva en las aulas (Moos y Ringdal, 2012). Los docentes consideran dedicar tiempo al trabajo sobre comprensión de textos, aunque la enseñanza explícita de estrategias aparece mínimamente cuando se analiza la actividad en el aula (Ness, 2016). Al abordar actividades de comprensión de textos en elaula, en general se repiten esquemas de participación simple: texto-preguntas (Sánchez-Miguel, 2016), que no consideran la enseñanza explícita de estrategias. En la misma línea, la escasa explicitación de estrategias metacognitivas por parte de los docentes queda señalada en la revisión de 17 estudios de observación en contextos de clase (Dignath y Veenman, 2020). Entre los docentes de primaria está presente el diseño de ambientes que estimulen la autorregulación pero es muy escaso el tiempo que dedican a enseñar estrategias en forma explicita (Dignath y Büttner, 2018). 
Por otra parte, en Uruguay los maestros demandan recursos y estrategias para acompañar a estudiantes con distintos niveles de lectura en sus aulas (Machado, 2019). Al mismo tiempo existe una baja probabilidad de que los estudiantes utilicen estrategias vinculadas al monitoreo, comprensión y uso de recursos personales en tareas complejas (INEEd, 2018). Debe considerarse que la autorregulación del aprendizaje no se desarrolla de forma espontánea sino que requiere instrucción directa, entrenamiento y práctica (Vandevelde, Van Keer y Merchie, 2016).

\section{Buscando intervenciones posibles y efectivas}

Retomando el metaanálisis realizado por Okkinga et al. (2018), de las estrategias enseñadas, definir las metas de la lectura fue la que tuvo un mayor efecto sobre la comprensión de textos. Asimismo, pensar en voz alta durante la lectura y explicitar sus procesos cognitivos parecen ser prácticas útiles para mejorar la habilidad estratégica.

En su metaanálisis, Ripoll y Aguado (2014) recogen 39 estudios que muestran que la enseñanza de estrategias de comprensión lectora combinadas con estrategias metacognitivas produce mejoras importantes en la comprensión lectora. Las actividades vinculadas al aprendizaje autorregulado son: la activación de conocimientos previos, de esquemas y conocimientos sobre la estructura textual; la supervisión de la propia comprensión a través del uso de organizadores gráficos, el automonitoreo y la autopregunta; el resumen como estrategia de evaluación. Ritchey et al. (2017) realizaron una intervención con 46 alumnos de $5^{\circ}$ grado con dificultades en comprensión de textos. En pequeños grupos y fuera del aula regular enseñaron explícitamente seis estrategias: realizar predicciones en base a títulos e imágenes, conectar con conocimientos previos, reparar si falla la comprensión, hacerse preguntas, explicar con sus propias palabras y evaluar si pueden contestar las preguntas que se formularon. Encontraron una diferencia significativa a favor del grupo experimental en el conocimiento y uso de estrategias para la comprensión y en comprensión de textos.

Rogiers, Merchie y Van Keer (2020a) implementaron un programa de enseñanza explícita de estrategias para aprender a través de la lectura, en el contexto real del aula con alumnos de $1^{\circ}$ año de secundaria. Los docentes participantes debian modelar estrategias, enseñarlas explícitamente, dar oportunidades para practicarlas y gradualmente ir disminuyendo la guía. Las estrategias enseñadas se dividieron en tres fases:

- Antes (escanear el texto, observar títulos e imágenes y preguntarse cuál es el tema del texto, preguntarse sobre sus conocimientos previos y plantearse objetivos).

- Durante (realizar una lectura global, resaltar las ideas principales, hacerse preguntas, parafrasear el contenido del texto, resumir, releer y vincular con conocimientos previos, monitorear el progreso, decidir cambiar de estrategia si es necesario y mantenerse en la tarea).

- Después de la lectura (preguntarse qué aprendi sobre este tema, qué hice bien, qué puedo mejorar y qué podría hacer de forma diferente la próxima vez).

Al finalizar la intervención los alumnos del grupo experimental lograron poner en práctica un repertorio de estrategias más amplio e integrado.

Entre los antecedentes de intervención en lengua castellana se encuentra el estudio de Fonseca et al. (2019) con escolares argentinos, en el que se incorporan estrategias de monitoreo y control de la comprensión de textos al trabajo del aula. 
Al considerar los antecedentes en su conjunto parece relevante continuar proponiendo y evaluando intervenciones en el aula basadas en la enseñanza explícita de estrategias de autorregulación en la comprensión de textos que involucren a los docentes y asuman las restricciones del aula.

Otro aspecto señalado es considerar qué beneficios ofrece ese tipo de intervenciones a los estudiantes con diagnóstico de dislexia. En definitiva, se trata de avanzar en el conocimiento que permita que todos los estudiantes logren aprendizajes de calidad (Alexander, 2018).

\section{Propósito del estudio}

El objetivo general del estudio es evaluar la incidencia de la enseñanza de estrategias de autorregulación del aprendizaje en la comprensión de textos escritos mediante una intervención educativa implementada en el aula, para apoyar el aprendizaje académico que realizan escolares de 5. año a partir de textos en el área de ciencias sociales. Particularmente, se espera determinar los efectos de la intervención en los estudiantes con diagnóstico de dislexia.

Se espera que los estudiantes que participan de la intervención enfocada en la enseñanza de autorregulación en comprensión de textos incrementen sus niveles de comprensión lectora y aumenten sus posibilidades de autorregulación (Okkinga et al., 2018). Del mismo modo, se espera que los estudiantes con diagnóstico de dislexia incrementen sus niveles de comprensión de textos y de autorregulación del aprendizaje (Sanders et al., 2019).

\section{Metodología}

\section{Participantes}

La muestra estuvo conformada por 69 estudiantes de $5 .{ }^{\circ}$ año de educación primaria de una institución privada de nivel socioeconómico medio-alto, en Montevideo, Uruguay (Ver Tabla 1). El 60.9\% eran varones y el 39.1\% eran niñas, de entre 10 y 11 años de edad. Veinte de los participantes (29\%) presentaban diagnóstico de dislexia, 14 de ellos en la condición experimental y seis en la de control.

La escuela se seleccionó por conveniencia, dadas las posibilidades para la intervención que la misma ofrecía y siendo lugar de trabajo de una de las autoras de este artículo. En forma aleatoria dos de los grupos de clase fueron asignados a la condición experimental, mientras que el grupo de clase restante fue considerado grupo de control. Asistieron al menos al 80\% de las sesiones de intervención. 
Tabla 1. Distribución de la muestra por sexo

\begin{tabular}{|l|l|l|l|}
\hline & Niñas & Varones & Total \\
\hline Grupo Experimental & $19(27.5 \%)$ & $\begin{array}{l}27 \\
(39.1 \%)\end{array}$ & $\begin{array}{l}46 \\
(66.6 \%)\end{array}$ \\
\hline Grupo Control & 8 & $\begin{array}{l}15 \\
(27.3 \%)\end{array}$ & $\begin{array}{l}23 \\
(38.9 \%)\end{array}$ \\
\hline Total & $(11.6 \%)$ & $\begin{array}{l}69 \\
(100 \%)\end{array}$ \\
\hline
\end{tabular}

\section{Diseño}

Se trata de un trabajo de investigación aplicada que asume las restricciones del aula (número de estudiantes, decisiones curriculares, momentos del curso escolar). Se presenta un estudio cuasi-experimental de diseño pre-post, con grupo de cuasi-control (Shadish, Cook y Campbell, 2002). Se considera como variable independiente la enseñanza de estrategias de autorregulación del aprendizaje, que varió en dos condiciones (experimental y control). Las variables dependientes son la comprensión de textos y la autorregulación del aprendizaje, y la eficacia lectora se considera como covariable.

\section{Instrumentos}

Se administró un cuestionario de comprensión de textos para evaluar la comprensión lectora. Se plantean 16 preguntas con modalidad múltiple opción a partir de la lectura de un texto expositivo (Balbi, Cuadro y Trías, 2009; Ramos y Cuetos, 2003). De las cuatro opciones solo una respuesta es la correcta. El acierto se contabiliza con un punto, con un máximo de 16. Estas preguntas demandan tareas de comprensión del texto base a nivel local y a nivel global, así como elaboración del modelo de situación. La fiabilidad de la prueba, analizada a través del alfa de Cronbach, resultó de .73 (Balbi et al., 2009). Se ha administrado a escolares uruguayos con fines de investigación (Ronqui, 2017; Trias, 2017).

Se utilizó la escala de gestión de la cognición de la prueba ARATEX-R (Núñez, Amieiro, Álvarez, García y Dobarro, 2015) para evaluar estrategias de autorregulación del aprendizaje implicadas en la comprensión de textos. Recoge estrategias contempladas en el modelo de autorregulación de Pintrich (2004). Se centra particularmente en la evaluación del monitoreo y supervisión que se realiza durante la tarea de lectura con el propósito de comprensión, y permite el ajuste de la propia actividad lectora. Este componente es especialmente relevante en la actividad de comprensión de textos. Se trata de un cuestionario de autoinforme al que los participantes deben responder pensando en lo que hacen cuando leen textos para aprender. Se compone de cuatro items tipo Likert, por ejemplo: "Para comprender bien un texto trato de unir la nueva información que me aporta con lo que ya sé sobre el tema". Su fiabilidad fue estimada mediante el alfa de Cronbach con un valor de .76 en la muestra del estudio. Para su utilización con escolares uruguayos se consultó previamente a dos maestras experimentadas y a las tres maestras de clase, quienes consideraron que los items se encontraban claramente redactados y su comprensión resultaría accesible a los estudiantes. Asimismo se realizó previamente un estudio piloto dirigido a evaluar el ajuste de los estudiantes a la consigna, sus posibilidades de respuesta y la calidad de los items. 
La prueba TECLE, que se encuentra adaptada, validada y baremada para escolares uruguayos (Cuadro, Costa, Trías y Ponce de León, 2009) se empleó con el fin de evaluar la eficacia lectora mediante la consideración de la precisión y la velocidad. Se considera un test de velocidad, que debe ser resuelto en 5 minutos. La puntuación directa es el número de respuestas correctas a partir de 64 ítems que contienen una frase encabezado y cuatro opciones de respuesta.

\section{Intervención}

El objetivo principal de la intervención es enseñar estrategias de autorregulación del aprendizaje al abordar textos de ciencias sociales en el contexto del aula. Se recurre al modelo cíclico de Zimmerman (2000) para identificar estrategias de autorregulación de aprendizajes, y a los aportes de Sánchez-Miguel (2016) sobre la enseñanza de estrategias de comprensión lectora.

Se trabaja sobre doce textos seleccionados del área de Ciencias Sociales, que siguen los lineamientos del programa escolar y que son considerados pertinentes por las docentes del curso. A medida que avanza la intervención aumenta la complejidad de los textos, considerando su extensión, la presencia de imágenes y cuadros explicativos.

En cada sesión en el aula se propone que la docente presente y modele una serie de estrategias de autorregulación del aprendizaje relevantes para la comprensión de textos. Luego se ofrecen espacios de trabajo compartido en los que los estudiantes pueden ensayar las estrategias y recibir retroalimentación a partir de su actividad. Por último, se proponen momentos de trabajo autónomo. Previo a la lectura, al presentar el texto y la tarea, se abordan estrategias correspondientes a la fase de planificación: activar conocimientos previos, establecer objetivos, definir resultados esperados, analizar la tarea e imaginar un plan de acción. Durante la lectura, en la fase de ejecución se aborda especialmente el monitoreo por medio de las preguntas como criterio para ir supervisando la acción y presentando alternativas ante eventuales dificultades. Se acompaña esta fase con la retroalimentación de la docente. Al cerrar la lectura, en la fase de evaluación, se orienta especialmente a reportar el proceso y las acciones realizadas durante la lectura. Cada sesión se cierra con una actividad de producción donde cada alumno da respuesta a las preguntas guía: desarrollo de respuestas breves, elaboración de un resumen, realización de esquemas y de mapas conceptuales.

\section{Procedimiento}

La docente encargada de la implementación dio comienzo con la planificación de la intervención en colaboración con los demás autores de este trabajo. En base a esta planificación se realizó un estudio piloto con un grupo de $5^{\circ}$ año de primaria de la cohorte anterior. Se pilotearon los instrumentos de evaluación y se observó particularmente la variabilidad de las medidas. Se pusieron a prueba tres sesiones de intervención, lo que sirvió como instancia de capacitación para quien implementaría dicha intervención. Se identificaron ejemplos de cómo presentar y modelar las estrategias de autorregulación, así como de posibles formas de retroalimentarlas. 
Antes (T1) y después (T2) de la intervención se evaluaron las variables dependientes mediante la administración colectiva del cuestionario de comprensión de textos y de la escala de gestión cognitiva. Se administró colectivamente la prueba TECLE en T1, considerada covariable. Se relevó la información disponible en la escuela para identificar a los niños con diagnóstico de dislexia, considerando los informes de técnicos especializados que lo certifican.

Luego de la evaluación en T1, los dos grupos de clase asignados a la condición experimental participaron de 12 sesiones de intervención. Las sesiones a cargo de una de las autoras del presente estudio implicaban una hora de trabajo en aula, en dos sesiones semanales. Esta intervención se desarrolló durante seis semanas, en la segunda mitad del año. Se tomó registro de asistencia, se recogieron documentos de planificación y cada sesión de trabajo fue grabada en audio para su posterior transcripción. Las tres primeras sesiones fueron supervisadas a partir de los registros de la docente encargada de implementar la intervención.

Paralelamente, el grupo control trabajó en el aula con los mismos textos siguiendo una estructura de participación directa (Sánchez-Miguel, 2016) en la que presentaba el texto y se realizaban preguntas sobre el mismo. Una vez realizada la evaluación en T2, el grupo de control recibió el mismo tratamiento experimental.

Durante la intervención se solicitó a las tres docentes de los grupos de clase convocados que continuaran trabajando en sus aulas tal como lo tenían previsto. Al finalizar se compartió con las docentes más detalles de los objetivos y las características de la intervención implementada.

El estudio contó con la autorización de la dirección general de la escuela y de las familias de los participantes.

\section{Fidelidad de la implementación}

Existen diversos criterios que pueden considerarse para analizar la fidelidad de la implementación, tales como: las bases teóricas, los documentos que presentan la intervención, su duración efectiva y frecuencia, su calidad, el modelo de intervención, los componentes críticos o la respuesta de los participantes (Century, Rudnick y Freeman, 2010; de Leeuw, de Boer y Minnaert, 2020).

La docente planificó previamente la intervención en el aula, la puso a prueba en el estudio piloto y, a partir de ello, realizó los ajustes que entendió más oportunos. Se desarrollaron las 12 sesiones que estaban previstas, abordando los contenidos y las actividades definidos previamente en el contexto del aula. Los participantes han asistido al menos al $80 \%$ de las sesiones y han realizado las actividades individuales correspondientes.

En cuanto a la presencia de los componentes criticos de la intervención, a partir de la transcripción de seis de las 12 sesiones, un observador externo analizó y categorizó las verbalizaciones de la docente (Nuñez, 2020). En estas sesiones se identificó la presencia de mensajes correspondientes a distintas fases del modelo cíclico de autorregulación (planificación, ejecución y evaluación). La fase de planificación fue la que estuvo más representada, con más del 40\% de las verbalizaciones categorizadas. Predominó la activación de conocimientos previos, la formulación de un plan de acción y el análisis de tareas. La fase de ejecución y evaluación variaron entre el 20 y 30\% de las verbalizaciones categorizadas en las distintas sesiones. Las verbalizaciones orientadas a revisar y repasar fueron las que predominaron en la fase de ejecución. En la fase de evaluación sobresalieron las verbalizaciones vinculadas al proceso. A partir del análisis de la actividad docente durante la intervención se confirmó la presencia de los componentes críticos de la intervención diseñada en base al modelo cíclico de Zimmerman (2000). 


\section{Análisis de datos}

Se utilizó el paquete estadístico SPSS versión 22. Inicialmente se obtuvieron los estadisticos descriptivos. A los efectos de comprobar la normalidad de las variables se realizó la prueba de Kolmogorov-Smirnov, con la corrección de significación de Lilliefors. Se utilizó ANOVA para comparar las medias de autorregulación y ANCOVA para las medias de comprensión de textos, considerando la eficacia lectora como covariable. Para estimar el tamaño del efecto se ha calculado eta cuadrado parcial $\left(\eta^{2}\right)$; considerando pequeño $\eta^{2}=.01$, medio $\eta^{2}=.06$, grande $\eta^{2}=$ .14 (Daily, Mann, Kristjansson, Smith \& Zullig, 2019). Para analizar los datos de los participantes con diagnóstico de dislexia dado su número reducido se utilizaron estadísticos no paramétricos: prueba de signos de Wilcoxon y prueba de MannWhitney. Se calculó $r$ como estimador del tamaño del efecto en las pruebas no paramétricas (Tomczak y Tomczak, 2014). El nivel de significación considerado ha sido $p<.05$

\section{Resultados}

En primer lugar se calcularon los estadísticos descriptivos de las medidas de comprensión de textos, autorregulación del aprendizaje y eficacia lectora (Ver Tabla 2). Se empleó la prueba de Kolmogorov-Smirnov con la corrección de significación de Lilliefors para comprobar la normalidad de las variables. Presentaron una distribución normal las variables CT-T2, con D(69) =.10, $p=.17$; GC$T_{1}$, con $D(69)=.10, p=.17$; GC-T2, con D(69) =.09, $p=.20$, y TEC-T1, con D(69) =.09, $p=.20$. La variable CT-T1 no se distribuye normalmente, con $D(69)=.18, p<.001$.

Luego se evaluó el efecto de la intervención en las estrategias de autorregulación mediante ANOVA, comparando grupo controly experimental en los dos momentos (T1 y T2). En esta medida de autorregulación se observó un efecto de interacción $\left(F_{(1.67)}=9.22, p<.00, \eta^{2}=.12\right)$ La incidencia de la intervención es significativa y se refleja en las estrategias de autorregulación vinculadas a la gestión cognitiva.

Se utilizó una prueba ANCOVA de medidas repetidas, considerando esta vez la medida de Comprensión de Textos (CT) como variable dependiente, el momento de evaluación (T1 y T2) y la condición (experimental y control) como variables independientes, y la eficacia lectora evaluada inicialmente como covariable. La covariable eficacia lectora resultó significativa $\left(F_{(1,66)}=5.98, p=.02, \eta^{2}=.08\right)$. También fueron significativos los efectos de la interacción $\left(F_{(1.66)}=17.90, p<.00, M^{2}=.21\right.$ ). La incidencia de la intervención resultó significativa y se reflejó en una mayor puntuación del grupo experimental en comprensión de textos en T2, considerando las puntuaciones de eficacia lectora. 
Tabla 2. Estadísticos descriptivos de Comprensión de textos, Autorregulación y Eficacia lectora

\begin{tabular}{|c|c|c|c|c|c|c|}
\hline & & \multicolumn{2}{|c|}{$\begin{array}{l}\text { Compresión de } \\
\text { textos }\end{array}$} & \multicolumn{2}{|c|}{ Autorregulación } & \multirow{2}{*}{$\begin{array}{l}\text { Eficacia } \\
\text { lectora }\end{array}$} \\
\hline & & $C T^{1}-T_{1}$ & CT-T2 & $G^{2}-T_{1}$ & GC-T2 & \\
\hline \multirow{4}{*}{$\begin{array}{l}\text { Total } \\
(n=69)\end{array}$} & M & 9.23 & 10.78 & 2.89 & 3.70 & 31.32 \\
\hline & DS & 2.68 & 2.69 & 0.84 & 0.79 & 10.61 \\
\hline & Min & 3.00 & 4.00 & 1.00 & 2.00 & 12.00 \\
\hline & Max & 14.00 & 16.00 & 4.25 & 5.00 & 56.00 \\
\hline \multirow{4}{*}{$\begin{array}{l}\text { Exper. } \\
(n=46)\end{array}$} & M & 8.80 & 11.15 & 2.79 & 3.92 & 30.74 \\
\hline & DS & 2.76 & 2.78 & 0.77 & 0.77 & 10.96 \\
\hline & Min & 3.00 & 6.00 & 1.50 & 2.25 & 12.00 \\
\hline & Max & 14.00 & 16.00 & 4.25 & 5.00 & 56.00 \\
\hline \multirow{4}{*}{$\begin{array}{l}\text { Exper--D4 } \quad(n= \\
14)\end{array}$} & $M$ & 8.00 & 9.79 & 2.64 & 4.07 & 21.64 \\
\hline & DS & 3.04 & 2.94 & 0.86 & 0.74 & 5.51 \\
\hline & Min & 3.00 & 6.00 & 1.50 & 2.50 & 12.00 \\
\hline & Max & 13.00 & 16.00 & 3.75 & 5.00 & 31.00 \\
\hline \multirow{4}{*}{$\begin{array}{l}\text { Control } \\
(n=23)\end{array}$} & M & 10.09 & 10.04 & 2.87 & 3.25 & 32.48 \\
\hline & DS & 2.33 & 2.40 & 0.97 & 0.64 & 10.01 \\
\hline & Min & 5.00 & 4.00 & 1.00 & 2.00 & 15.00 \\
\hline & Max & 13.00 & 12.00 & 4.25 & 4.25 & 52.00 \\
\hline \multirow{4}{*}{$\begin{array}{l}\text { Control-D } 5 \text { (n } \\
=6)\end{array}$} & M & 9.67 & 8.17 & 2.38 & 2.67 & 23.17 \\
\hline & DS & 2.58 & 3.06 & 1.13 & .52 & 9.00 \\
\hline & Min & 6.00 & 4.00 & 1.00 & 2.00 & 15.00 \\
\hline & Max & 13.00 & 12.00 & 4.25 & 3.25 & 39.00 \\
\hline
\end{tabular}

Nota: ${ }^{1}$ Cuestionario de comprensión de textos. ${ }^{2}$ Escala de gestión cognitiva. ${ }^{3}$ TECLE: Test de eficacia lectora. ${ }^{4}$ Niños con diagnóstico de dislexia en la condición experimental. ${ }^{5}$ Niños con diagnóstico de dislexia en la condición de control.

Considerando solamente a los participantes con diagnóstico de dislexia de ambas condiciones se realizaron comparaciones utilizando estadísticos no paramétricos. Se comparó la evolución de cada grupo respecto a sí mismo en las variables dependientes usando la prueba de signos de Wilcoxon. Al considerar la comprensión de textos, los niños con diagnóstico de dislexia del grupo experimental aumentaron sus puntuaciones en T2 $(M d n=9.50)$, aunque no resultó significativa su variación respecto a $T_{1}(M d n=7.50), z=-1.70, p=.09, r=-.32$. Tampoco fue significativa la leve disminución del grupo control en T2 $(M d n=9.00)$ respecto a $T_{1}(M d n=9.50), z=-1.19, p=.24, r=-.34$. En cuanto a la autorregulación, los participantes con diagnóstico de dislexia en la condición experimental aumentaron significativamente su puntuación en gestión cognitiva en T2 ( $M d n=$ 4.13) respecto a $T_{1}(M d n=2.75), z=-3.18, p<.01, r=-.60$. No se observaron cambios significativos en autorregulación de los niños con dislexia en el grupo control en $\mathrm{T}_{2}(\mathrm{Mdn}=2.63)$ respecto a $\mathrm{T} 1(\mathrm{Mdn}=2.38), z=-.84, p=.40, r=-.24$

Finalizada la intervención (T2), los participantes con diagnóstico de dislexia del grupo experimental $(M d n=4.13)$ alcanzaron una puntuación significativamente mayor en gestión cognitiva que sus pares del grupo control (Mdn $=2.63)$, al compararlos mediante la prueba de Mann-Whitney, $U=6.50, z=-2.94, p<.01, r=$ -.46 . 


\section{Discusión y conclusiones}

En primer lugar se destaca que la intervención basada en la enseñanza explícita de estrategias de autorregulación del aprendizaje impacta positivamente tanto en la comprensión de textos como en la autorregulación. Este resultado se alinea con la evidencia disponible, que sostiene que las estrategias de autorregulación se pueden enseñar de forma explícita y que ello contribuye de forma positiva al aprendizaje de la comprensión de textos (Dignath y Büttner, 2008; Okkinga et al., 2018). Se trata de una relación relevante si se pretende avanzar en intervenciones educativas que mejoren los desempeños de los estudiantes en la comprensión de textos (INEEd, 2018).

Asimismo, el efecto en la comprensión de textos es esperable, y se observa en textos informativos que se trabajan habitualmente en ese nivel escolar en el área de ciencias sociales. Estos mejores desempeños a la hora de comprender textos van más allá de esta intervención focalizada y constituyen una herramienta para seguir aprendiendo a partir de los textos, lo que resulta clave en el sistema educativo (Kim, 2015). No significa que todos los estudiantes resuelvan de forma excelente las tareas de comprensión de textos, claro está, sino que poseen más estrategias para afrontarlas y mejorar sus desempeños.

En este caso, el efecto de la intervención es significativo en comprensión de textos al controlar los niveles de eficacia lectora. La posibilidad de decodificar de forma acertada y fluida se relaciona con el aprovechamiento de la intervención, dada la relación que existe entre las variables (Balbi et al., 2009). El control de la eficacia lectora importa en la medida en que los grupos no se han conformado al azar, y el grupo cuasi-control no necesariamente resulta equivalente. Por tanto, habiendo medido la eficacia lectora, se sostiene el efecto de la intervención sobre la comprensión de textos.

En la misma línea se observa un efecto positivo de la intervención con respecto a la autorregulación del aprendizaje. Luego de haber participado de la intervención los estudiantes afirman que tienen más presentes las estrategias de gestión cognitiva muy vinculadas a la comprensión de textos, tales como relacionar ideas durante la lectura, detectar las ideas centrales y vincularlas o relacionar las ideas previas con la nueva información leída. Se trata de estrategias estrechamente relacionadas con la tarea de comprensión de textos, que permiten orientar el monitoreo, contribuir al control de la actividad y evaluarla de modo que permita seguir aprendiendo. Estas estrategias están presentes en la propuesta de intervención implementada. De todos modos, este aumento significativo en las puntuaciones de autorregulación puede reflejar mayor conciencia de los estudiantes y con ello más posibilidades de autorregulación, o la adquisición del conocimiento declarativo que les permite decir lo que hacer pero no necesariamente hacerlo. La convergencia con el resultado en comprensión de textos orienta a pensar que refleja un modo distinto de involucrarse y autorregular las actividades de comprensión de textos.

En segundo lugar es de destacar que estos efectos se observan a partir de una intervención implementada en el contexto del aula, asumiendo las dinámicas propias delámbito escolar (contenidos, propósitos, número de estudiantes, formas de interacción, distracciones, etc.) y ya no en un contexto más controlado como un laboratorio. La explicitación de las estrategias de autorregulación enriquece la estructura de participación directa que habitualmente se presenta en clase para actividades de comprensión de textos (Sánchez-Miguel, 2016). Se trata de un aspecto relevante de esta investigación ya que se parte de la premisa de que la enseñanza de la autorregulación aún no se ha extendido en las aulas (Dignath y Veenman, 2020; Ness, 2016). 
Las evidencias de fidelidad de la intervención aportan solidez a los resultados y permiten caracterizar de mejor modo la intervención (de Leeuw, de Boery Minnaert, 2020). En este caso, llevar adelante la intervención ha implicado una oportunidad de formación en servicio para la maestra: acceso a teoría e investigación sobre la temática, filtrado de esos contenidos a partir de su experiencia en el aula, selección de las estrategias a abordar y finalmente aplicación de esa planificación en su dinámica de clase. Probablemente, proponer explícitamente las estrategias de autorregulación en clase implica desarrollar la autorregulación del propio docente (Kramarski, 2018). La selección de varias estrategias a partir del modelo ciclico de Zimmerman (2013) enriquece la actividad docente y a la vez permite ajustarse a una actividad tan compleja como es la comprensión de textos. Si bien la intervención implementada hace foco en las estrategias de autorregulación, no puede dejar de considerarse el clima que ello requiere y las instancias de reflexión metacognitiva que van más allá de las estrategias tanto para los estudiantes como para los docentes.

En tercer lugar, la intervención se ha realizado en el aula con grupos heterogéneos donde conviven niños con diversas características y perfiles, entre ellos, alumnos con diagnóstico de dislexia. La inclusión de estos alumnos es un desafío permanente para la labor educativa y, a la vez, una tarea demandada por investigaciones previas (Sanders et al., 2019). Al considerar los efectos solamente en los niños con diagnóstico de dislexia que participaron de la intervención se observa un aumento en su puntuación en autorregulación, es decir, mayor conciencia de sus procesos de gestión cognitiva y quizá mayor utilización de estas estrategias. Este incremento brinda mayores posibilidades de control durante la tarea que, de mantenerse, puede contribuir a su rendimiento académico a futuro. Sin embargo, el aumento de sus puntuaciones en comprensión de textos no resulta estadisticamente significativo. Para lograr un efecto significativo en la comprensión de textos en estos niños con diagnóstico de dislexia deberian contemplarse con más detenimiento algunas estrategias que les permitieran afrontar posibles dificultades que la lectura puede generarles, dificultades relacionadas al vocabulario, la fatiga y los aspectos afectivos que la lectura moviliza. Enriquecer la intervención considerando alguno de estos aspectos podría resultar beneficioso para el conjunto de los estudiantes. Asimismo, podría incrementarse la intensidad de la intervención, sumando algunas sesiones o breves tareas domiciliarias.

El reducido tamaño de la muestra es uno de los límites del presente trabajo, lo que afecta particularmente la comparación de niños con diagnóstico de dislexia. A la vez, contar con el diagnóstico de dislexia no describe la situación de los participantes ni el estado de sus procesos lectores al momento del estudio. Se entiende como un aporte la inclusión de estos niños. Así como se avanza en la inclusión educativa, se debería continuar profundizando en el conocimiento de los efectos de distintas intervenciones educativas y de sus requerimientos.

Si bien el uso de los instrumentos es ajustado a los propósitos y alcance de la investigación, la evaluación de procesos tan complejos como la comprensión de textos y el aprendizaje autorregulado es otro de los límites que debe señalarse. A la hora de evaluar la comprensión de textos hay falta de consenso y se encuentran dificultades dada la multiplicidad de procesos que la componen (Calet, López-Reyes y Jiménez-Fernández, 2019). Es señalado que la evaluación de la autorregulación es uno de los desafíos más importantes para la investigación en este campo (Panadero, Klug y Järvelä, 2015). Particularmente, al utilizar autoinformes, los estudiantes de educación primaria pueden presentar dificultades para generalizar cuál es su forma típica de afrontar la tarea, y pueden tener un sesgo positivo. Por lo tanto, en futuras intervenciones podrian triangularse medidas de autorregulación que se obtienen a partir de autoinformes con otras herramientas de evaluación tales como el análisis del pensamiento en voz alta y rastros de la actividad (Heirweg et al., 2019; Rogiers, Merchie y Van Keer, 
2020b). Futuros trabajos deberian considerar la evaluación intentando tomar en cuenta la complejidad de los procesos implicados en la autorregulación en la comprensión de textos, los requisitos metodológicos de las buenas medidas y los usos educativos de las mismas. Seguramente la combinación de enfoques cuantitativos y cualitativos será bienvenida para atender ese desafío.

Uno de los desafios a atender en futuros trabajos deriva de las posibilidades y demandas que enfrentan los estudiantes al aprender en múltiples textos y con la mediación digital. Leer textos en contexto digital exige mayor autorregulación a los estudiantes (Burin et al., 2020), por lo que sostener la actividad a través de la acción educativa se vuelve muy relevante.

Sin dudas, uno de los principales desafios a futuro es crecer en el involucramiento de los docentes en la investigación sobre autorregulación. Esto permitirá construir conocimientos más pertinentes y útiles para la tarea educativa en la promoción de procesos de aprendizaje y desarrollo. A la vez permitirá extender los beneficios de la enseñanza de la autorregulación del aprendizaje al trabajo en aula.

Esta intervención muestra que implementar estrategias de autorregulación del aprendizaje enfocadas en comprensión lectora resulta beneficioso para los estudiantes en su conjunto y puede realizarse en modalidades compatibles con las dinámicas, exigencias y necesidades propias del trabajo en el aula. Constituye un camino posible para avanzar en una educación de calidad, que permita seguir aprendiendo a lo largo de la vida y abarque a todos los estudiantes.

Nota: Aprobación final del artículo, editora responsable Mag. Verónica Zorrilla de San Martín.

Contribución de autoria: Todos los autores participaron de forma equitativa en las etapas de diseño de la investigación, recolección de datos, procesamiento, análisis y elaboración del texto. 


\section{Referencias bibliográficas}

ALEXANDER, P. A. (2018). Looking down the Road: Future Directions for Research on Depth and Regulation of Strategic Processing IMirando hacia el futuro: futuras direcciones para la investigación sobre la profundidad y la regulación del procesamiento estratégico]. British Journal of Educational Psychology, (88), 152-166. https://doi.org/10.1111/bjep.12204

BALBI, A., CUADRO, A. Y TRÍAS, D. (2009). Comprensión lectora y reconocimiento de palabras. Ciencias Psicológicas, III(2), 153-160. http://www.scielo.edu.uy/ scielo.php?pid=S1688-42212009000200004\&script=sci_arttext

BURIN, D. I., GONZÁLEZ, F. M., BARREYRO, J. P., \& INJOQUE-RICLE, I. (2020). Metacognitive Regulation Contributes to Digital Text Comprehension in E-learning IContribuciones de la regulación metacognitiva a la comprensión de textos digitales en el E-learningl. Metacognition and Learning. https://doi. org/10.1007/s11409-020-09226-8

CALET, N., LÓPEZ-REYES, R., \& JIMÉNEZ-FERNÁNDEZ, G. (2019). Do Reading Comprehension Assessment Tests Result in the same Reading Profile? A Study of Spanish Primary School Children l¿Las pruebas de evaluación de comprensión lectora dan como resultado el mismo perfil de lectura? Un estudio de niños españoles de educación primarial. Journal of Research in Reading, 43. 98-115. https://doi.org/10.1111/1467-9817.12292

CENTURY, J., RUDNICK, M., \& FREEMAN, C. (2010). A Framework for Measuring Fidelity of Implementation: A Foundation for Shared Language and Accumulation of Knowledge IUn marco para medir la fidelidad de la implementación: una base para el lenguaje compartido y la acumulación de conocimientos]. American Journal of Evaluation, 31(2), 199-218. https://doi.org/10.1177/1098214010366173

CUADRO, A., COSTA, D., TRIAAS, D. Y PONCE DE LEÓN, P. (2009). Manual técnico del test de eficacia lectora (TECLE). Prensa Médica Latinoamericana.

DAILY, S. M., MANN, M. J., KRISTJANSSON, A. L., SMITH, M. L., \& ZULLIG, K. J. (2019). School Climate and Academic Achievement in Middle and High School Students Students IClima escolar y rendimiento académico en estudiantes de secundaria y bachillerato]. Journal of School Health, 89(3), 173-180. https://doi. org/10.1111/josh.12726

DE BOER, H., DONKER, A. S., KOSTONS, D. D. N. M., \& VAN DER WERF, G. P. C. (2018). Long-term Effects of Metacognitive Strategy Instruction on Student Academic Performance: A Meta-analysis [Efectos a largo plazo de la enseñanza de estrategias metacognitivas sobre el rendimiento académico de los estudiantes: un metaanálisis]. Educational Research Review, 24(February), 98-115. https://doi. org/10.1016/j.edurev.2018.03.002

DE LEEUW, R. R., DE BOER, A. A., \& MINNAERT, A. E. M. G. (2020). The Proof of the Intervention Is in the Implementation; ASystematic Reviewabout Implementation Fidelity of Classroom-Based Interventions Facilitating Social Participation of Students with Social-Emotional Problems or Behavioural Difficulties [La prueba de la intervención está en la implementación; una revisión sistemática sobre la fidelidad de la implementación de las intervenciones en el aula que facilitan la participación social de los estudiantes con problemas socioemocionales o dificultades de comportamiento]. International Journal of Educational Research Open, 1, 100002. https://doi.org/10.1016/j.ijedro.2020.100002

DENT, A. L., \& KOENKA, A. C. (2016). The Relation Between Self-Regulated Learning and Academic Achievement across Childhood and Adolescence: A MetaAnalysis ILa relación entre la autorregulación del aprendizaje y el rendimiento académico en la infancia y la adolescencia: un metaanálisis]. Educational Psychology Review, 28(3), 425-474. https://doi.org/10.1007/s10648-015-9320-8 
DIGNATH, C., BUETTNER, G., \& LANGFELDT, H.-P. (2008). How can primary school students learn self-regulated learning strategies most effectively?: A Meta-analysis on Self-regulation Training Programmes l¿Cómo pueden los estudiantes de primaria aprender estrategias de autorregulación del aprendizaje de manera más efectiva?: un metaanálisis sobre programas de enseñanza de la autorregulación]. Educational Research Review, 3(2), 101-129. https://doi. org/10.1016/j.edurev.2008.02.003

DIGNATH, C., \& BÜTTNER, G. (2008). Components of Fostering Self-regulated Learning among Students. A Meta-analysis on Intervention Studies at Primary and Secondary School Level. IComponentes de la promoción del desarrollo autorregulado en los estudiantes. Un metaanálisis de estudios de intervención en la educación primaria y secundaria]. Metacognition and Learning, 3(3), 231264. https://doi.org/10.1007/s11409-008-9029-x

DIGNATH, C., \& BÜTTNER, G. (2018). Teachers' Direct and Indirect Promotion of Self-Regulated Learning in Primary and Secondary School Mathematics Classes - Insights from Video-Based Classroom Observations and Teacher Interviews [Enseñanza directa e indirecta del aprendizaje autorregulado en las clases de matemáticas de la escuela primaria y secundaria: datos de observaciones de grabaciones de clases y entrevistas con maestros]. Metacognition and Learning, (April). https://doi.org/10.1007/s11409-018-9181-X

DIGNATH, C., \& VEENMAN, M. V. J. (2020). The Role of Direct Strategy Instruction and Indirect Activation of Self-Regulated Learning - Evidence from Classroom Observation Studies IEl papel de la enseñanza directa y la activación indirecta de la autorregulación del aprendizaje - evidencia de estudios de observación en el aula]. Educational Psychology Review. https://doi.org/10.1007/s10648-02009534-0

FONSECA, L., MIGLIARDO, G., SIMIAN, M. Y OLMOS, R. (2019). Estrategias para mejorar la comprensión lectora: impacto de un programa de intervención en español. Psicología Educativa, 25(2), 91-99. https://doi.org/10.5093/psed2019a1

FONSECA, L., PUJALS, M., LASALA, E., LAGOMARSINO, I., MIGLIARDO, G., ALDREY, A., BUONSANTI, L. Y BARREIRO, J. P. (2014). Desarrollo de habilidades de comprensión lectora en niños de escuelas de distintos sectores socioeconómicos. Revista Neuropsicología Latinoamericana, 6(1), 41-50. https:// www.redalyc.org/articulo.oa?id $=439542506005$

GAETA, M. L. (2015). Aspectos personales que favorecen la autorregulación del aprendizaje en la comprensión de textos académicos en estudiantes universitarios. Revista de Docencia Universitaria, 13(2), 17-35. https://doi. org/10.4995/redu.2015.5436

GREENE, J. A. (2018). Self-regulation in education [La autorregulación en la educación]. Routledge. https://doi.org/10.4324/9781315537450

HEIRWEG, S., DE SMUL, M., DEVOS, G., \& VAN KEER, H. (2019). Profiling upper Primary School Students' Self-Regulated Learning Through Self-Report Questionnaires and Think-Aloud Protocol Analysis ICreando perfiles de aprendizaje autorregulado en los estudiantes del último ciclo de primaria a través de cuestionarios de autoinforme y análisis de protocolos de pensamiento en voz alta]. Learning and Individual Differences, 70(December 2017), 155-168. https://doi.org/10.1016/j.lindif.2019.02.001

INEED (2018). Marco de habilidades socioemocionales en sexto de educación primaria. Montevideo: INEEd. https://www.ineed.edu.uy/images/Aristas/ Publicaciones/Marcos/Aristas-Marco-HabilidadesSocioemocionales-Primaria. pdf 
KIM, Y.-S. (2015). Language and Cognitive Predictors of Text Comprehension: Evidence from Multivariate Analysis [Predictores cognitivos y del lenguaje de la comprensión de textos: evidencia de análisis multivariado]. Child Development, 86(1), 128-144. https://doi.org/10.1111/cdev.12293

KRAMARSKI, B. (2018). Teachers as Agents in Promoting Students' SRL and Performance. Applications for Teachers' Dual-role Training Program [Los docentes como agentes en la promoción de la autorregulación del aprendizaje y el desempeño de los estudiantes: aplicaciones del programa de formación de doble función de los docentes]. In D. H. Schunk \& J. A. Greene (Eds.), Handbook of self-regulation of learning and performance (2 $2^{\text {nd }}$ ed., 223-239). Routledge. https://doi.org/10.4324/9781315697048-15

MACHADO, P. (2019). Necesidades técnico-pedagógicas para la enseñanza de la lectura: voces de maestros noveles. RELAPAE, 10, 22-37. https://revistas.untref. edu.ar/index.php/relapae/article/view/226

MOOS, D. C., \& RINGDAL, A. (2012). Self-Regulated Learning in the Classroom: A Literature Review on the Teacher's Role ILa autorregulación del aprendizaje en el aula: una revisión sobre el rol de los docentes]. Education Research International, 2012, 1-15. https://doi.org/10.1155/2012/423284

NESS, M. (2016). Reading Comprehension Strategies in Secondary Content Area Classrooms: Teacher Use of and Attitudes towards Reading Comprehension Instruction [Estrategias de comprensión de textos en las áreas de contenidos en secundaria: prácticas y actitudes de los docentes hacia la enseñanza de la comprensión de textos]. Reading Horizons: A Journal of Literacy and Language Arts, 55(1).

NÚÑEZ, J. C., AMIEIRO, N., ÁlVAREZ, D., GARCÍA, T. Y DOBARRO, A. (2015). Escala de Evaluación de la Autorregulación del Aprendizaje a partir de Textos (ARATEX-R). European Journal of Education and Psychology, 8(1), 9-22. https:// doi.org/10.1016/j.ejeps.2015.10.002

NUÑEZ, R. (2020). Autorregulación del aprendizaje y comprensión de textos: análisis de una práctica de aula [Trabajo de fin de grado no publicado]. Universidad Católica del Uruguay.

OKKINGA, M., VAN STEENSEL, R., VAN GELDEREN, A. J. S., VAN SCHOOTEN, E., SLEEGERS, P., \& ARENDS, L. R. (2018). Effectiveness of Reading-Strategy Interventions in Whole Classrooms: a Meta-Analysis [Efectividad de las intervenciones en estrategias de lectura en el aula: un metaanálisis]. Educational Psychology Review, (30), 1215-1239. https://doi.org/10.1007/s10648-018-9445-7

PANADERO, E. (2017). A Review of Self-regulated Learning: Six Models and Four Directions for Research IUna revisión del aprendizaje autorregulado: seis modelos y cuatro direcciones para la investigación]. Frontiers in Psychology, 8(April), 1-28. https://doi.org/10.3389/fpsyg.2017.00422

PANADERO, E., KLUG, J., \& JÄRVELÄ, S. (2015). Third Wave of Measurement in the Self-Regulated Learning Field: When Measurement and Intervention come Hand in Hand ITercera ola de evaluación en el campo del aprendizaje autorregulado: cuando la evaluación y la intervención van de la mano]. Scandinavian Journal of Educational Research, (August), 1-13. https://doi.org/10.1080/00313831.2015 .1066436

PERRY, J., LUNDIE, D., \& GOLDER, G. (2018). Metacognition in Schools: What does the Literature Suggest about the Effectiveness of Teaching Metacognition in Schools? [Metacognición en las escuelas: ¿Qué sugiere la literatura sobre la efectividad de la enseñanza de la metacognición en las escuelas?]. Educational Review, (April), 1-18. https://doi.org/10.1080/00131911.2018.1441127 
PINTRICH, P. R. (2004). A Conceptual Framework for Assessing Motivation and SelfRegulated Learning in College Students IUn marco conceptual para evaluar la motivación y el aprendizaje autorregulado en estudiantes de educación superior]. Educational Psychology Review, 16(4), 385-407. https://doi.org/10.1007/ \$10648-004-0006-X

RAMOS, J. L. Y CUETOS, F. (2003). PROLEC-SE. TEA.

RIPOLL, J. C. Y AGUADO, G. (2014). La mejora de la comprensión lectora en español: un metaanálisis. Revista de Psicodidáctica/Journal of Psychodidactics, 19(1), 2744. https://doi.org/10.1387/RevPsicodidact.9001

RITCHEY, K. D., PALOMBO, K., SILVERMAN, R. D., \& SPEECE, D. L. (2017). Effects of an Informational Text Reading Comprehension Intervention for FifthGrade Students IEfectos de una intervención en comprensión de textos para estudiantes de quinto grado de educación primarial. Learning Disabilities Quarterly, 40(2), 68-80. https://doi.org/10.1177/0731948716682689

ROGIERS, A., MERCHIE, E., \& VAN KEER, H. (2020). Learner Profile Stability and Change over Time: The Impact of the Explicit Strategy Instruction Program "Learning Light" [Estabilidad y cambios en el tiempo del perfil de aprendizaje: el impacto del programa de enseñanza explícita "learning light"]. Journal of Educational Research, 113(1), 26-45. https://doi.org/10.1080/00220671.2019.171 1005

ROGIERS, A., MERCHIE, E., \& VAN KEER, H. (2020). Opening the Black Box of Students' Text-Learning Processes: A Process Mining Perspective [Abriendo la caja negra de los procesos de aprendizaje a través de textos de los estudiantes: una perspectiva desde el "process mining"]. Frontline Learning Research, 8(3), 40-62. https://doi.org/10.14786/flrv8i3.527

RONQUI, V. (2017). El papel del lenguaje y la autorregulación en comprensión de textos según el nivel socioeconómico. [Tesis de Maestría no publicada]. Universidad Católica del Uruguay.

SÁNCHEZ-MIGUEL, E. (2016). Aspiraciones y conocimientos: ¿cómo explicar que la innovación educativa resulte decepcionante? El caso de la comprensión lectora. Álabe, 7(13), 1-22. http://revistaalabe.com/index/alabe/article/view/348

SANDERS, S., LOSINSKI, M., PARKS ENNIS, R., WHITE, W., TEAGARDEN, J., \& LANE, J. (2019). A Meta-Analysis of Self-Regulated Strategy Development Reading Interventions to Improve the Reading Comprehension of Students With Disabilities IUn metaanálisis de intervenciones en el desarrollo de estrategias de autorregulación para mejorar la comprensión lectora de estudiantes con discapacidades]. Reading and Writing Quarterly, 35(4), 339-353. https://doi.org/1 $\underline{0.1080 / 10573569.2018 .1545616}$

SHADISH, W. R., COOK, T. D., \& CAMPBELL, D. T. (2002). Experimental and QuasiExperimental Designs for Generalized Causal Inference IDiseños experimentales y cuasi-experimentales para inferencia causal generalizada]. Houghton Mifflin. https://www.alnap.org/system/files/content/resource/files/main/147.pdf

STEINER, M., VAN LOON, M. H., BAYARD, N. S., \& ROEBERS, C. M. (2020). Development of Children's Monitoring and Control when Learning from Texts: Effects of Age and Test Format [Desarrollo del monitoreo y control en niños en el aprendizaje a través de textos: efectos de la edad y el formato de la evaluación]. Metacognition and Learning, (15), 3-27. https://doi.org/10.1007/ s11409-019-09208-5 
TOMCZAK, M., \& TOMCZAK, E. (2014). The Need to Report Effect Size Estimates Revisited. An Overview of Some Recommended Measures of Effect Size [La necesidad de informar las estimaciones del tamaño del efecto. Una descripción general de algunas medidas recomendadas del tamaño del efecto]. Trends in Sport Sciences, 1(21), 19-25. https://www.researchgate.net/ publication/303919832_The_need_to_report_effect_size_estimates_revisited_ An_overview_of_some_recommended_measures_of_effect_size

TRIAS, D. (2017). Autorregulación en el aprendizaje, análisis de su desarrollo en distintos contextos socioeducativos. [Tesis doctoral no publicada]. Universidad Autónoma de Madrid.

UNESCO (2016). Declaración de Incheon para la Educación 2030 y Marco de Acción Hacia una educación inclusiva y equitativa de calidad y un aprendizaje a lo largo de la vida para todos. http://www.unesco.org/new/fileadmin/MULTIMEDIA/ FIELD/Santiago/pdf/ESP-Marco-de-Accion-E2030-aprobado.pdf

VANDEVELDE, S., VAN KEER, H., \& MERCHIE, E. (2016). The Challenge of Promoting Self-Regulated Learning among Primary School Children with a Low Socioeconomic and Immigrant Background [El desafío de promover el aprendizaje autorregulado entre los niños de primaria migrantes y de bajo nivel socioeconómico]. Journal of Educational Research, 110(2), 113-139. https://doi.or g/10.1080/00220671.2014.999363

ZEIDNER, M., \& STOEGER, H. (2019). Self-Regulated Learning (SRL): A guide for the perplexed [Autorregulación del aprendizaje: una guía para los desconcertados]. High Ability Studies, 30:1-2, 9-51, https://doi.org/10.1080/13598139.2019.1589369

ZIMMERMAN, B. J. (2013). From Cognitive Modeling to Self-Regulation : A Social Cognitive Career Path [Del modelado cognitivo a la autorregulación: una trayectoria profesional cognitiva sociall. Educactional Psychologist, 48(3), 135147. https://doi.org/10.1080/00461520.2013.794676

ZIMMERMAN, B. J. (2000). Attaining Self-regulation. A Social Cognitive Perspective [Lograr la autorregulación. Una perspectiva socio-cognitiva]. In M. Boekaerts, P. Pintrich, \& M. Zeidner (Eds.), Handbook of SelfRegulation. Academic Press. https://books.google.com.uy/books?hl=en $\& l r=\& i d=r v 3 D Z \operatorname{Sim} 6 z 4 C \& 0 i=f n d \& p g=P P 1 \& d q=I n+M+$ Boekaerts, + P. + Pintrich +\%26+M.+Zeidner+(Eds.), +Handbook+of+Self-Regulation.+San+Diego:+Academic+Press.\&ots=tovaCbtWSi\&sig=ZNJgbaT97UUQRkcXrx5CDUIDcIM\&redir esc $=y \# v=$ onepage \&q=In\%20M.\%20Boekaerts\%2C\%20P.\%20Pintrich\%2C\%20 $\% 26 \% 20$ M.\%20Zeidner\%20(Eds.)\%2C\%20Handbook\%20of\%20SelfRegulation.\%20San\%20Diego\%3A\%20Academic\%20Press.\&f=false 